\title{
GEOPLOT: UM SISTEMA GRÁFICO PARA REPRESENTAÇŌES DE MODELOS DIGITAIS DE TERRENOS
}

\author{
J.K.Yamamoto
}

\section{RESUMO}

Este trabalho apresenta um sistema gráfico, denominado GEOPLOT, desenvolvido para obter representações gráficas espaciais de modelos digitais de terrenos. GEOPLOT é um conjunto de programas escritos em linguagenm TURBO-PASCAL, para microcomputadores nacionais compativeis ao IBM-PC. Estes programas permitem obter o mapa de localização de pontos, a fronteira convexa de um conjunto de pontos de dados, a interpolaçăo do modelo digital do terreno e suas representações gráficas (i.e., mapa de contorno e o bloco-dlagrama). Foi desenvolvido também um programa que permite gerar as saldas gráficas dos programas do GEOPLOT diretamente na impressora matricial de pontos. Finalmente, o sistema gráfico GEOPLOT proporciona representações gráficas de modelos digitais de terrenos com rapidez, precisão e versatilidade.

\section{ABSTRACT}

This paper presents a graphics system, designated GEOPLOT, developed to display digital terrain models graphically. GEOPLOT is a set of TURBO PASCAL programs for IBM-PC compatible microcomputers. These programs allow one to obtain a map of a data points, the convex hull of a set of data points, and the interpolation of a the digital model and its graphical displays (l.e. contour map and perspective plot). This system also contains a computer program that retrieves the

\footnotetext{
'Departamento de Geologia Econరิmica e Geofisica Aplicada, Instituto de Gecciências/USP.
} 
plotting files from display programs and puts out high quality drawings directly on standart dot printers. Finally GEPLOT graphics system provides graphical displays of digital terrain models rapidly, with accuracy and versatility.

\section{INTRODUÇĀO}

Este trabalho apresenta um sistema de computação gráfica desenvolvido especialmente para obtençăo de representaçöes de modelos digitais de terrenos.

Os modelos digitals de terrenos representam a topografia de uma determinada região, através de medidas de elevaçőes feitas sobre um arranjo regular de pontos. Quando os pontos não estão em arranjo regular, procede-se ao ajuste de funções matemáticas que são utilizadas para calcular as elevações sobre os nós de uma malha topologicamente regular, que constitui então o modelo digital do terreno.

Assim, dado o modelo digital do terreno, pode-se obter as representações gráficas mais comuns, quais sejam: mapas de contomos e blocos-dlagrama. Toda a metodologia básica para a obtençăo de representaçöes gráficas de modelos digitais de terrenos auxilladas por computador está descrita em YAMAMOTO (1988).

\section{COLOCAÇÃO DO PROBLEMA}

As representaçőes gráficas de modelos digitais de terrenos podem ser obtidas através de programas de computaçăo com grandes vantagens, tais como: rapidez, precisão $\theta$ versatilidade. Para que isso seja possivel, é necessário um conjunto de programas que permitem determinar as representaçőes gráficas, que săo entăo dirigidas para os dispositivos de salda gráfica. Tais programas, quando desenvolvidos $\theta$ instalados em microcomputadores nacionais, tornam-se compativeis e portáteis. Quanto aos dispositivos e saída, estes podem ser: monitor de video, plotter ou impressora matricial de pontos. Os monitores de video permitem a visualização de desenho dentro da limitação da dimensão e resolução gráfica da tela. É possivel obter-se uma cópia do desenho da tela (hardcopy) na impressora matricial de pontos, por meio de um programa do sistema operacional em disco. Os traçadores digitais de desenhos, plotters, recebem comandos gráficos padrões, os quais são reproduzidos no papel utilizando pequenos segmentos de reta. Finalmente, as impressoras 
matricials de ponto também poderiam reproduzir desenhos, não como hardcopy limitada à resolução da placa de vídeo, mas sim com a resoluçăo dela própria, que é bem superior às placas de vídeo existentes no mercado. Para isso é necessário um programa de computação, o qual receba como entrada os comandos gráficos de plotagem, que normalmente seriam dirigidos para o plotter, $\theta$ os reproduza na impressora programando as agulhas da cabeça previamente ao avanço da mesma. Em

outras palavras, esse programa faz a conversăo de um processo vetorial para raster. É preciso ressaltar que a resolução gráfica de desenhos em impressoras, embora superior à maioria das placas de video do mercado, é inferior àquela dos plotters. Como consequência dessa implementação, temse na impressora matricial de pontos um desenho de alta resolução e qualidade gráfica a custos reduzidos, pois pode substituir em parte o plotter que ainda tem um custo muito elevado no país. Uma outra vantagem desse programa é que torna os sistemas de computação gráfica totalmente portátels podendo ser utilizados no campo sem a necessidade de transportar plotters.

O sistema de computação gráfica GEOPLOT é composto por esse programa, justamente para ser utilizado no campo por pesquisadores da área das Geociências.

\section{DESCRIÇÃO DO SISTEMA GEOPLOT}

O sistema de computaçăo gráfica GEOPLOT foi desenvolvido especialmente para obtenção de representações de modelos digitais de terrenos. Esse sistema é composto por vários programas todos escritos em linguagem TURBO PASCAL, para microcomputadores nacionais de 16 bits, conforme o fluxograma operacional da Figura 1.

Tal fluxograma considera o caso mais geral em que os dados de elevação do terreno estão distribuidos irregularmente na reglăo de Interesse e, portanto, necessitam da interpolação para determinação do modelo digital do terreno. Se os pontos de dados já estiverem sobre os nós de uma malha topologicamente regular, o processamento pode ser iniciado diretamente da etapa de representações gráficas de modelos digitals de terrenos.

Assim, considerando o caso mais geral, o processamento é iniciado com o desenho do mapa de localização dos pontos de dados, através do programa LOCALPAS. O mapa de localização é muito útil na definiçăo do método de interpolação a ser utilizado, bem como da abertura ótima da malha regular do modelo digital do terreno.

Numa fase seguinte, independente da anterior, os mesmos pontos de dados são utilizados para determinação da fronteira convexa dos dados, que é o polígono convexo de área 


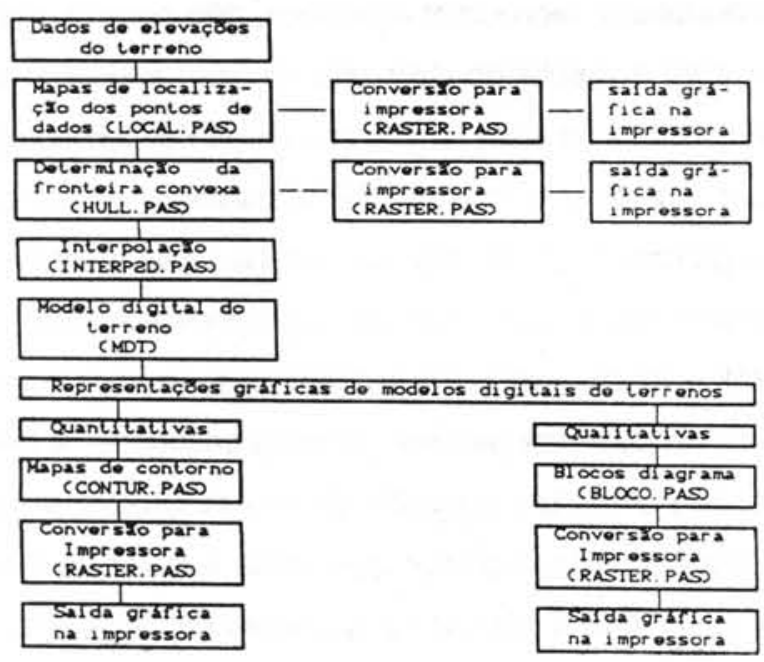

Figura 1 - Fluxograma operacional do sistema GEOPLOT.

mínima que engloba os dados. A fronteira convexa dos dados é utilizada para definir o domínio dentro do qual a interpolação será efetuada, ou seja, serão interpolados somente os nós que caem dentro da mesma. O algorftmo utilizado no programa HULLPS é aquele publicado por EDDY (1977) em FORTRAN IV e adaptado para linguagem TURBO PASCAL

Determinada a fronteira convexa dos dados e definida a abertura da malha regular, o programa de interpolação INTERP2D.PAS reconhece os nós que pertencem ao domínio da fronteira convexa, através de um algorttmo publicado na literatura especializada por HAL (1975). Esse algorftmo baseia-se na soma orientada dos ângulos entre o nó da malha regular a ser interpolado e os vértices do poligono convexo; quando esta soma for $\pm 360^{\circ}$, significa que o nó pertence à fronteira convexa e quando for $0^{\circ}$, significa que o nó esta fora.

O programa INTERP2D.PAS usa as equações multiquádricas ajustadas sobre suportes locais como método de interpolação, de acordo com a descrição apresentada em YAMAMOTO (1988).

Obtido o modelo digital do terreno, procede-se às representações gráficas propriamente ditas. Tais representações podem ser de natureza quantitativa ou qualitativa, respectivamente, mapa de contorno e bloco-dlagrama.

Os mapas de contorno representam as intersecções entre os planos de determinadas elevações com a superfícle do terreno. Tais intersecções são obtidas pelo programa CONTUR.PAS, pesquisando-se os pontos do modelo digital do terreno usando o método do rastreamento (YAMAMOTO, 1988). 
O programa BLOCO.PAS faz a projeção em perspectiva da malha regular de dados, segundo formulação proposta em KUBERT et al. (1968). As linhas ocultas ao observador, em função da projeção em perspectiva, são eliminadas aplicando-se o algorftmo de WRIGHT (1973).

Finalmente, o programa RASTER.PAS, utilizado em todas as saídas gráficas do sistema GEOPLOT, transforma os comandos gráficos do arquivo de plotagem em pontos na impressora matricial de pontos. Esse programa é compatf́vel com todas as impressoras nacionais que seguem o padrão EPSON, entre outras: EMIUA PC, GRAFIX, MONICA PLUS e RIMA XT-180. Todos os comandos gráficos de plotagem, gerados pelos demais programas de Sistema GEOPLOT, são gravados em arquivos tipo texto (ASC II).

A resolução gráfica resultante é de 120 pontos por polegada na horizontal por 144 pontos por polegada na vertical, isto é, densidade dupla em ambas as direções. A área útil de desenho é igual a $30,48 \mathrm{~cm}$ (12 polegadas) no eixo horizontal $X$ por $25,4 \mathrm{~cm}$ (10 polegadas) no eixo vertical $Y$. Todas as coordenadas são recebidas $\mathrm{em} \mathrm{cm}$, tendo como origem o canto inferior esquerdo.

O programa RASTER.PAS reconhece seis pseudo-códigos de plotagem, conforme formato e descrição dos mesmos a seguir:

- código 0

formato: [x y o]

efeito: muda a origem atual do sistema de coordenadas para aquela especificada em $(x, y)$;

- código 1

formato: [x y 1 fator]

efeito: aplica um fator escala (fator) especificado a todos os dados dimensionais lidos a partir deste comando;

\section{- código 2}

formato: [x y 2]

efeito: move a "pena" da posição em que se encontra até a posição de coordenadas $(x, y)$ desenhando, ou seja, com a pena abaixada (no início do processamento a "pena" encontra-se na origem); 
- código 3

formato: [x y 3]

efeito: move a "pena" da posição em que se encontra até a posição de coordenadas $(x, y)$ sem desenhar, ou seja, com a pena levantada;

- código 4

formato: [x y 4 alt angle $~$ string ]

efelto: escreve um conjunto de caracteres entre dois sinais de (til), com altura da letra igual a alth, em cm, a partir da posição de coordenadas $(x, y)$ e ângulo igual a angle, em graus decimais, medldo no sentido anti-horário, a partir do ebxo $X$;

- código 9

formato: [x y 9 ]

efelto: fecha o arquivo de plotagem. Após esse comando o desenho gravado na memória do computador é enviado à impressora.

\section{EXEMPLO DE UTILIZAÇÃO DO SISTEMA GEOPLOT}

Para llustrar uma aplicação típica do sistema GEOPLOT, fol utilizado um conjunto de pontos de dados amostrados aleatoriamente de uma área de $10 \times 10 \mathrm{Km}$ da folha topográfica de Cunha, editada pelo IBGE.

Os pontos amostrados, onde foram lidas as cotas do terreno sobre a carta topográfica de Cunha, bem como a fronteira convexa dos mesmos, encontram-se desenhados na Figura 2. Isto foi feito inserindo-se o arquivo de saida do programa HULLPLAS no arquivo do programa LOCALPAS, através de um processador de textos. Dessa forma é possivel, em qualquer etapa do processamento, inserir arquivos dentro de outros para obter desenhos compostos.

O modelo digital de terreno fol obtido calculando-se as elevações do terreno para os nos pertencentes à fronteira convexa, através do ajuste local de equações multiquádricas. Para esse exemplo o modelo foi representado por $21 \times 21$ nós.

Os pontos do modelo digital do terreno assim calculados são então processados pelos programas de representações gráficas, quais sejam: CONTUR.PAS e BLOCO.PAS. O mapa de 
contorno gerado pelo programa CONTUR.PAS encontra-se reproduzido no desenho da Figura 3 , enquanto na Figura 4 tem-se representada a projeção em perspectiva do modelo digital do terreno para um observador situado a $150^{\circ}$ de azimute.

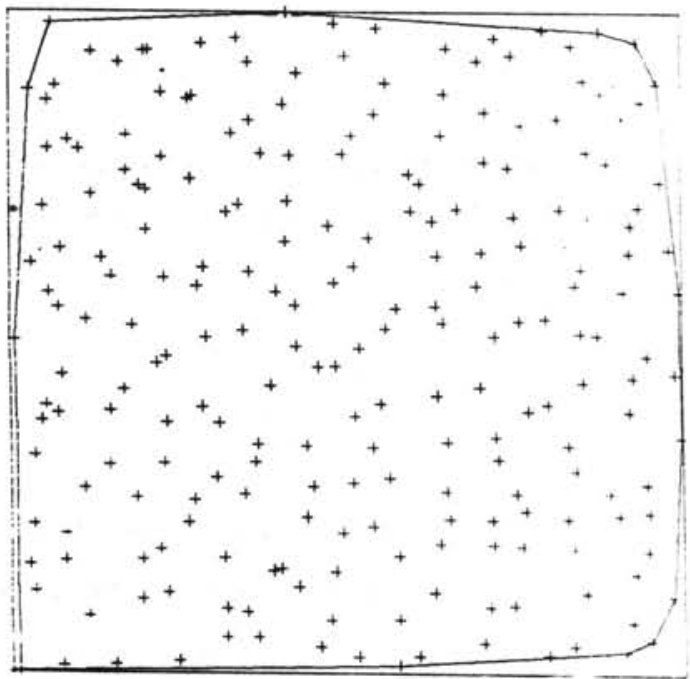

Figura 2 - Mapa de localizaçăo dos pontos de dados com desenho da fronteira convexa. Este desenho é resultado dos processamentos nos programas LOCALPAS e HULLPAS, cujos arquivos foram editados para um único por meio de um processador de textos.

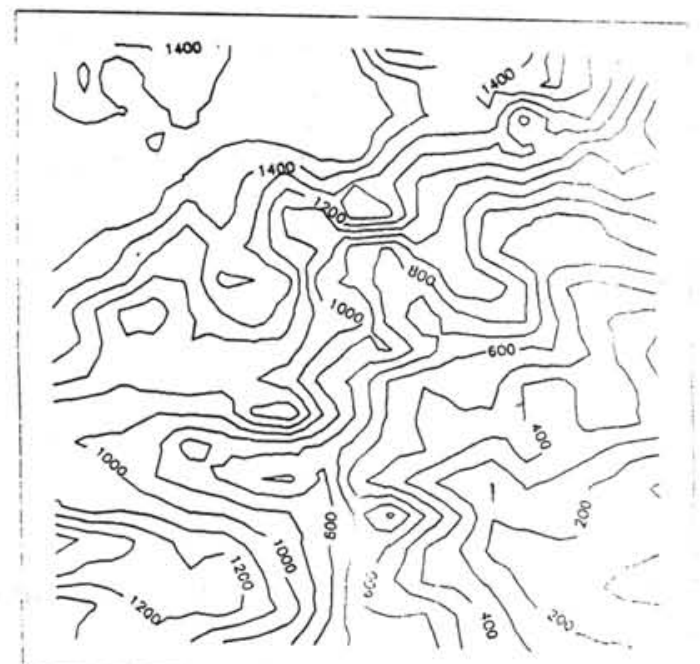

Figura 3 - Mapa de contorno do modelo digital do terreno, obtido por ajuste local de equaçóes multiquádricas em uma malha regular de 21 por 21 nós. O mapa apresenta-se com a borda truncada evido à eliminaçăo dos nós năo pertencentes à fronteira convexa durante o processo da interpolaçăo (programa INTERP2D.PAS). Este desenho é resultado do processamento do programa CONTUR.PAS sobre o arquivo de saida do programa INTERP2D.PAS. 


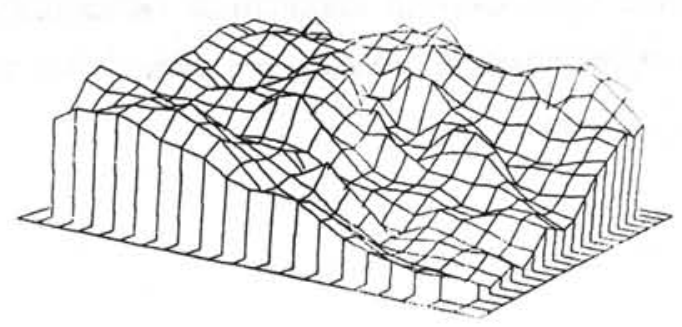

Figura 4 - Projeçăo em perspectiva do modelo digital do terreno para um observador situado a $150^{\circ}$ de azimute. Este blocodiagrama fol obtido do processamento do programa BLOCO.PAS para a malha regular interpolada (saida do programa INTERP2D.PAS).

\section{CONSIDERAÇÓES FINAIS}

Fol apresentado um sistema gráfico portátll, escrito em linguagem TURBO PASCAL, para microcomputadores de 16 bits e impressoras matriciais de pontos nacionais.

O sistema gráfico GEOPLOT proporciona representaçőes gráficas de modelos digitais de terrenos com rapidez, precisão e versatilidade.

O programa RASTER.PAS vem preencher uma lacuna em termos de software gráfico nacional, pois permite a obtenção de desenhos de alta resolução e qualidade diretamente na impressora matricial de pontos, eliminando, em parte, a necessidade imediata de "plotters". É preciso ressaltar que este programa não é único no seu gênero, pois existem atualmente vários sistemas, dessa natureza, disponfveis comerclalmente. Entretanto, o desenvolvimento desse programa permitiu ao autor o conhecimento das técnicas da computação gráfica utilizadas para esse fim. Uma outra vantagem desse desenvolvimento $e$ que tendo o programa fonte, este pode ser modificado segundo as necessidades especfficas dos usuários.

\section{REFERÊNCIAS BIBUOGRÁFICAS}

EDDY, W.F. (1977) A new convex hull algorithm for planar sets. ACM Transactions on Mathematical Software, 3(4):398-403.

HALL, J.K. (1975) PTLOC-A FORTRAN subroutine for determining the position of a point relative to a closed boundary. Mathematical Geology, 7(1):75-79 
HUBERT, B.; SZABO J.; GIULERI, S. (1968) The perspective representation of functions of two variables. Journal of ACM, 15(2):193-204.

WRIGHT, T.J. 1973) A two space solution to the hidden line problem for plotting functions of two variables. IEEE Transactions on Computers, C-22(1):28-23.

YAMAMOTO, J.K. (1988) Representaçöes gráficas de modelos digitais de terrenos. In: CONGRESSO NACIONAL DE INFORMÁTICA, 21., Rio de Janeiro, 1987. Anais. Rio de Janeiro, SUCESU, v.1, p.924-929. 